\title{
Outcome of Childhood Acute Lymphoblastic Leukemia Treated Using the modified-CCG-1881 and CCG-1882 Protocols at Pediatric Center of Hue Central Hospital, Vietnam
}

Chau Van Ha ${ }^{1}$, Pham Nhu Hiep ${ }^{2}$, Tran Kiem Hao ${ }^{1}$, Phan Xuan Mai ${ }^{1}$, Phan Huy Thuan ${ }^{1}$, Nguyen Thi Kim Hoa $^{1}$, Nguyen Dac Luong ${ }^{1}$, Watanabe Kazuyo ${ }^{3}$ and Nguyen Huu Son*1

${ }^{1}$ Pediatric Center, Hue Central Hospital, Vietnam

${ }^{2}$ Hue Central Hospital, Vietnam

${ }^{3}$ Asian Children's Care League

*Corresponding author: Nguyen Huu Son, Pediatric Center, Hue Central Hospital. 16 Le Loi street, Hue City, Vietnam

\section{ARTICLE INFO}

Received: 幽 April 24, 2019

Published: May 08, 2019

Citation: Chau Van H, Tran Kiem H, Phan Xuan M, Phan Huy T, Nguyen Thi Kim H, Nguyen Huu S, Watanabe K, Pham Nhu H. Outcome of Childhood Acute Lymphoblastic Leukemia Treated Using the modified-CCG-1881 and CCG-1882 Protocols at Pediatric Center of Hue Central Hospital, Vietnam. Biomed J Sci \& Tech Res 17(5)-2019. BJSTR. MS.ID.003063.

Keywords: Childhood Acute Lymphoblastic Leukemia; Complete Remission; Relapse; Treatment

Abbreviations: CCG: Children's Cancer Group; HCH: Hue Central Hospital; ALL: Acute Lymphoblastic Leukemia; SR: standard Risk; HR: High Risk; CR: Complete Remission; BM: Bone Marrow; CNS: Central Nervous system; OS: Overall Survival; EFS: Event Free survival; AML: Acute Myeloid Leukemia
ABSTRACT

Background: Hue Central Hospital (HCH) plays a key role to treat Acute Lymphoblastic Leukemia (ALL) in the central zone of Vietnam which covers geographically wide areas. Before 2007, the survival rate was very low, and abandonment rate was more than $50 \%$. The aims of this study are to determine the outcome of newly diagnosed children with ALL treated at HCH from June 2007 to December 2017 and to report our experiences in reducing the abandonment.

Methods: This is a retrospective review of 238 children with ALL admitted from June 2007 to December 2017. The diagnosis was confirmed by morphological FAB criteria and cytochemistry. Patients were classified as standard risk (SR) or high risk (HR) according to NCI criteria, using modified-CCG-1881 and CCG-1882 protocol. Social supports were provided to patients/families.

Results: A total of 238 children with ALL were analyzed with mean age of 4.7 years ranging from 1 month to 16 years. The male to female ratio was 2:1. SR and HR patients were $139(59 \%)$ and 99 (41\%) respectively. The overall complete remission (CR) rate on day 28 of induction were $92.1 \%$ (SR) and $84.9 \%$ (HR). Induction death were $3.6 \%$ (SR) and $10.1 \%$ (HR). Patients accounted for $44(18.5 \%)$ had relapses which occurred in isolated $B M n=12$, isolated CNS $n=17$, combined $B M$ and CNS $n=13$, isolated testis $n=2$. Overall survival (OS) at 10 years were $70.7 \%$ (SR) and $51.5 \%$ (HR). The event-free survival (EFS) at 10 years were $66.1 \%(\mathrm{SR})$ and $47.3 \%(\mathrm{HR})$. Abandonment cases were 9 (3.8\%).

Conclusion: With less toxic modified protocol, survival rate has been improved and treatment related mortality was minimized though high relapse rate is still an issue. Abandonment has been reduced successfully with holistic strategies such as financial support, managing family group, providing education, early follow-up of patients who missed appointments and free accommodation near hospital for patients/families.

\section{Introduction}

Acute leukemia is the most common form of cancer in children comprising approximately 30 percent of all childhood malignancies [1]. Of the acute leukemias, acute lymphoblastic leukemia (ALL) occurs five times more commonly than acute myeloid leukemia (AML). Survival rates for ALL have improved dramatically since the 1980s, with a current five-year overall survival rates estimated at greater than 90 percent [2-5]. This improvement in survival is due to treatment of a large number of children on sequential standardized research protocols. Approximately 75 to 80 percent of children with newly diagnosed ALL participate in such trials, the 
goals of which are to improve clinical outcomes while minimizing acute toxicities and late-occurring adverse events. ALL is a heterogeneous disease; subtypes differ with regard to biological, cellular and molecular characteristics, response to therapy and risk of relapse, and are associated with different outcomes [6]. In order to standardize treatment of leukemia, we have completely applied the protocols modified-CCG-1881 and CCG-1882 for childhood leukemia treatment. Protocols were sub-classified according to the subtype of leukemia and clinical risk factors of patients at presentation. The aims of this study are to determine the outcome of newly diagnosed children with ALL treated at $\mathrm{HCH}$ and to report our experiences in reducing the abandonment.

\section{Materials and Methods}

This retrospective study was based on a sample of 238 under 15-year-old patients with newly diagnosed ALL treated with the modified CCG-1881 and CCG-1882 between June 2007 anđ December 2017. Patients were treated at the Pediatric Oncology department of Hue Central Hospital. All parents and/or patients gave their written informed consent. The study was approved by the Research Ethics Committees of Hue Central Hospital.

The observed clinical variables included age at diagnosis, gender, WBC count, hemoglobin and lactate dehydrogenase (LDH) levels, platelet count, immunophenotype. Treatment response was evaluated based on the presenting WBC count, the lymphoblast count on the eighth day of induction therapy (Day 8), and bone marrow (BM) analysis on Day 28 of induction.

ALL was diagnosed in patients with $\geq 25 \%$ lymphoblasts in BM (based on morphological and cytochemical evaluations of BM smears) and positivity in immunophenotyping and cytogenetics. The DI was determined by flow cytometry. Peripheral blood, bone marrow and cerebrospinal fluid (CSF) were collected at referral hospitals. According to the NCI risk criteria, risk of ALL (standard vs. high) was stratified based on age and WBC count at diagnosis. Standard-risk patients are in age less than one or greater than nine and WBC $>50.0 \times 09$ cells/L. And the remain population is in high-risk group. The statistical analysis was carried out using SPSS 20.0. Descriptive statistics were used to characterize the patients. Associations between variables, prognostic factors and response were analyzed with the chi-square test and Fisher's exact test. Fiveyear overall survival (OS) and event-free survival (EFS) rates were estimated with the Kaplan-Meier method and compared with the log-rank test. The Cox proportional hazards model was used to identify independent prognostic factors with respect to EFS and OS. The level of statistical significance was set at 5\% ( $p$-value <0.05).

\section{Results}

\section{Patient Characteristic}

We enrolled 238 children with ALL. Boys were more common than girls (2.0:1). The mean age was 4.7 years (range 1-15 years).
Standard risk and high risk patients were 139 (59\%) and 99 (41\%) respectively (Table 1). The median initial white blood cell count (WBC) was $12,800 / \mathrm{mm}^{3}$ and CD10 was positivity in $95 \%$ (Table 2).

Table 1: Patient characteristics.

\begin{tabular}{|c|c|c|}
\hline Variables & Number & $\%$ \\
\hline Age & & \\
\hline$<1$ & 4 & 1.7 \\
$1-<9$ & 178 & 74.8 \\
$>9$ & 56 & 23.5 \\
\hline Gender & & \\
\hline Male & 159 & 66.8 \\
\hline Female & 79 & 33.2 \\
\hline Risk group & & 58.8 \\
\hline Standard & 140 & 41.2 \\
\hline High & 98 & \\
\hline
\end{tabular}

Table 2: Baseline laboratory characteristics.

\begin{tabular}{|c|c|}
\hline Baseline Laboratory Characteristics & All Patients \\
\hline Hemoglobin $(\mathrm{g} / \mathrm{dL})$ & $8.24 \pm 2.68$ \\
\hline $\mathrm{WBC} \times 10^{9}$ cells/L & $12.8 \pm 3.35$ \\
\hline Platelets $\times 10^{9}$ cells $/ \mathrm{L}$ & $109.80 \pm 122.92$ \\
\hline LDH level (U/L) & $1506.43 \pm 1194.03$ \\
\hline CD10 positivity n (\%) & $226(95)$ \\
\hline
\end{tabular}

\section{Results of Treatment}

Most (89.1\%) of ALL patients achieved complete remission after induction. The respective remission rates were $92.1 \%$ and $84.9 \%$ for the standard risk and high risk. The death rates before completed remission were higher for high risk ALL compared to standard risk ALL (10.1\% vs 3.6\%). Of the $18.5 \%$ of ALL patients who relapsed. Relapses of ALL patients were mostly in CNS (12.6\%) while bone marrow relapses were 10.5\%; and there were 2 cases of testicular repapse $(0.8 \%)$ (Table 3 ).The overall survival and Event-free survival of ALL treated by modified CCG-1881 and CCG1882 protocols were significant higher in SR group compared to HR group (Figures 1 \& 2).

Table 3: Results of treatment.

\begin{tabular}{|c|c|c|c|}
\hline $\begin{array}{c}\text { Result of ALL } \\
\text { treatment }\end{array}$ & All Patients & SR Group & HR Group \\
\hline $\begin{array}{c}\text { Death before complete } \\
\text { induction }\end{array}$ & $15(6.3 \%)$ & $5(3.6 \%)$ & $10(10.1 \%)$ \\
\hline Complete remission & $212(89.1 \%)$ & $128(92.1 \%)$ & $84(94.9 \%)$ \\
\hline Relapse & $44(18.4 \%)$ & & \\
\hline CNS relapase & $17(7.1 \%)$ & & \\
\hline BM relapase & $12(5.0 \%)$ & & \\
\hline BM + CNS relapase & $13(5.5 \%)$ & & \\
\hline Testicular relapase & $2(0.8 \%)$ & & \\
\hline Treatment abandonment & $9(3.8 \%)$ & & \\
\hline
\end{tabular}




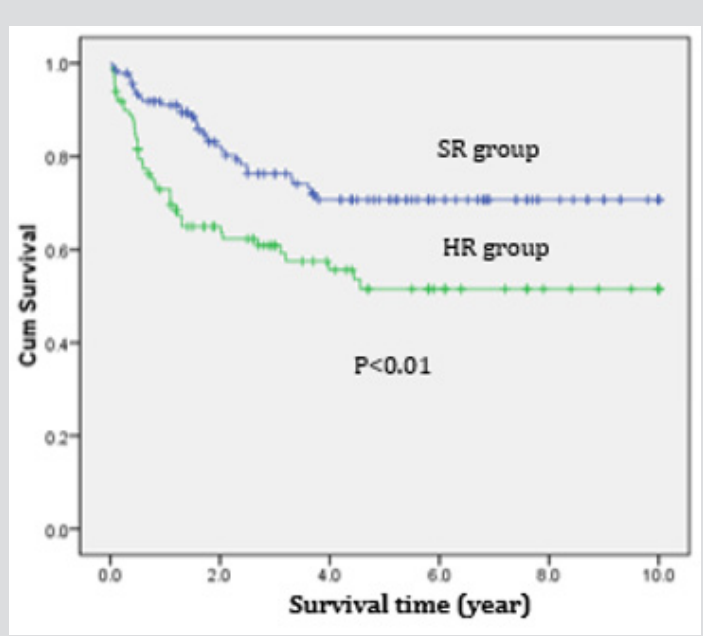

Figure 1: Overall Survival in Children with Acute Lymphoblastic Leukemia.

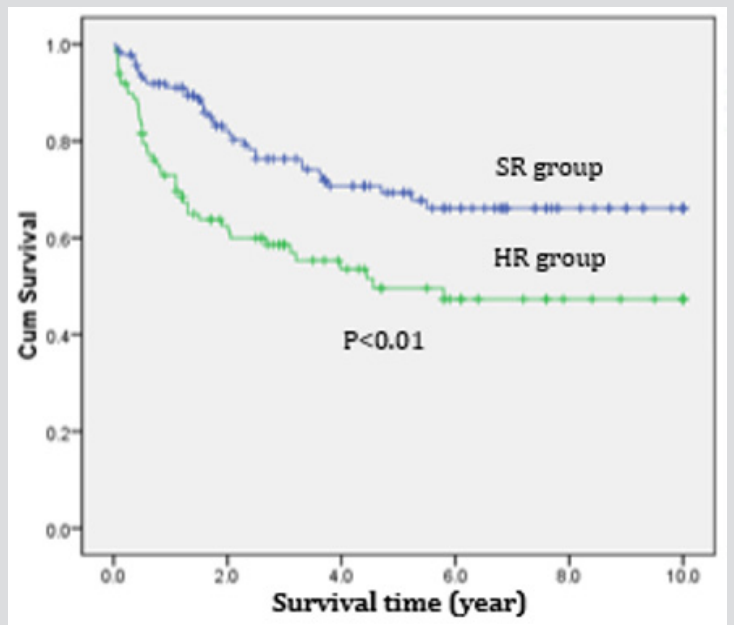

Figure 2: Event-free survival in Children with Acute Lymphoblastic Leukemia.

\section{Discussion}

The current 5-year survival rate of childhood acute lymphoblastic leukemia (ALL) exceeds $85 \%$ in developed countries. This improvement has been achieved by the optimal use of antileukemic agents, dose modification, improved drug combinations, optimized administration schedules, and better supportive care. As most patients with newly diagnosed ALL are cured, current studies are focused on curing relapsed or refractory patients, as well as the reduction of long-term treatment-related morbidity for survivors. A study at a tertiary care centre in Asia documented a relapse rate of $24 \%$ [7], whereas in this study, the relapse rate was lower (18.4). However, a study in the USA revealed a relapse rate of $16 \%$ [8]. Thus, although the relapse rate obtained in this study was better than that observed in other Asian studies, it was still inferior to that observed in developed countries. Therefore, further research and re-evaluation are required to identify the reason, to ensure better patient outcomes. Furthermore, in studies carried out in the USA and Asia $[7,8], \mathrm{BM}$ was the most common site of relapse, which is similar to the results of this study. In Asia, over two-thirds of ALL cases could be categorised as high-risk. In contrast, high-risk disease accounts for only 10\%-15\% of the ALL cases in developed countries [9-11]. In this study, high-risk disease was observed in $36 \%$ of the cases. The higher percentage of high-risk disease in this study compared with that observed in developed countries might be due to late referrals, but the inherent difference in the biology of leukaemia cells needs to be investigated. The high incidence of induction deaths $(6.3 \%$ overall, $\mathrm{n}=15 ; 3.6 \%$ in SR patients, and $10.1 \%$ in HR patients) was mostly because of infectious complications. This result is different from those of studies conducted in developing countries such as Vietnam where infection was the most common cause of death [12], while in developed countries such as the USA, the pre-B ALL type was the leading cause of death in children as well as in adults $[13,14]$.

The main reasons for these variations are differences in treatment, hospital resources, quality of infection control and socioeconomic status between developing and developed countries [12]. To assess whether anthracyclines played a role, we investigated the cumulative doses administered in patients who died during induction and observed that they had received a very limited amount. This was consistent with the protocol guidelines, which prescribed anthracyclines only to patients in good clinical condition. Thus, it appears that earlier diagnosis and better management of infectious complications may be more important than changes in chemotherapy during induction therapy. The best way to achieve a better outcome for childhood ALL treatment is to improve compliance to treatment through an effective comprehensive program that includes:

a) Parental education;

b) Family affective management;

c) A patient tracking system; and

d) Social services for families (i.e., transportation, food and lodging subsidies).

To meet international standards and improve treatment outcomes, we have completely applied the protocols modified CCG1881 and CCG-1882 for childhood leukemia treatment.

The incidence of treatment abandonment has been reduced to $3.8 \%$ in the current study. This was obtained thanks to a strong intervention by Asian Children's Care League, which was able to classify the living conditions of patients at the time of diagnosis and to provide support, including a family food bag, money for travel, housing for parents, and other support as needed, together with a program for parent's education to improve their understanding of the disease, special care needs, administration of oral chemotherapy, etc. This result can be regarded as an exceptional achievement and compares favorably with other contemporary experiences $[15,16]$. The overall results from this study suggest 
that intensive therapy can be delivered in a well organized center in an low-middle-income countries and that treatment abandonment can be reduced to a very low incidence with promising results. However, these results remain suboptimal because of the socioeconomic conditions that are associated with a higher risk of late diagnosis and early death. Longer follow-up also is needed to determine whether a plateau at 5 years has been reached with this therapy. Further improvement in survival should be pursued through educational programs to facilitate earlier diagnosis, better management of infectious complications, better knowledge of the disease, and possibly different treatment strategies.

\section{Conclusion}

Treatment protocols of modified-CCG-1881 and CCG-1882 to treat childhood ALL was successfully adapted and suggest that such an approach may be useful in other low- and middle-income countries.

\section{Conflicts of Interest}

The authors declare no conflicts of interest.

\section{References}

1. Svendsen AL, Feychting M, Klaeboe L, Langmark F, Schuz J (2007) Time trends in the incidence of acute lymphoblastic leukemia among children 1976-2002: a population-based Nordic study. J Pediatr 151(5): 548-550.

2. Al-Nasser A, El-Solh H, De Vol E, El-Hassan I, Alzahrani A, et al. (2008) Improved outcome for children with acute lymphoblastic leukemia after risk-adjSusted intensive therapy: a single-institution experience. Ann Saudi Med 28(4): 251-259.

3. De Moerloose B, Suciu S, Bertrand Y, Mazingue F, Robert A, et al. (2010) Improved outcome with pulses of vincristine and corticosteroids in continuation therapy of children with average risk acute lymphoblastic leukemia (ALL) and lymphoblastic non-Hodgkin lymphoma (NHL) report of the EORTC randomized phase 3 trial 58951. Blood 116(1): 3644.

4. Kolenova A, Kaiserova E, Makohusova M, Subova Z, Bubanska E, et al. (2015) Improved outcome for children and adolescent with acute lymphoblastic leukemia in the first decade of the $21^{\text {st }}$ century: A report from the Slovak Republic. Neoplasma 62(5): 812-820.

5. Pui CH, Sandlund JT, Pei D, Campana D, Rivera GK, et al. (2004) Improved outcome for children with acute lymphoblastic leukemia: results of Total Therapy Study XIIIB at St Jude Children's Research Hospital. Blood 104(9): 2690-2696.

6. Pui CH, Robison LL, Look AT (2008) Acute lymphoblastic leukemia. Lancet 371(9617): 1030-43.

7. Kulkarni KP, Marwaha RK, Trehan A, Bansal D (2009) Survival outcome in childhood ALL: experience from a tertiary care centre in North India. Pediatr Blood Cancer 53(2): 168-173.

8. Vora A, Frost L, Goodeve A, Wilson G, Ireland RM, et al. (1998) Late relapsing childhood lymphoblastic leukemia. Blood 92(7): 2334-2347.

9. Campana D, Behm FG (2000) Immunophenotyping of leukemia. J Immunol Methods 243(1-2): 59-75.

10. Evans WE, Relling MV, Rodman JH, Crom WR, Boyett JM, et al. (1998) Conventional compared with individualized chemotherapy for childhood acute lymphoblastic leukemia. N Engl J Med 338(8): 499-505.

11. Rivera GK, Raimondi SC, Hancock ML, Behm FG, Pui CH, et al. (1991) Improved outcome in childhood acute lymphoblastic leukaemia with reinforced early treatment and rotational combination chemotherapy. Lancet 337(8733): 61-66.

12. Asim M, Zaidi A, Ghafoor T, Qureshi Y (2011) Death analysis of childhood acute lymphoblastic leukaemia; experience at Shaukat Khanum Memorial Cancer Hospital and Research Centre, Pakistan. J Pak Med Assoc 61(7): 666-670.

13. Hossain MJ, Xie L, McCahan SM (2014) Characterization of pediatric acute lymphoblastic leukemia survival patterns by age at diagnosis. J Cancer Epidemiol: 865979.

14. Pui CH, Ribeiro RC (2003) International collaboration on childhood leukemia. Int J Hematol 78(5): 383-389.

15. De Pernillo M, Rivas S, Fuentes L, Antillon F, Barr RD (2014) Measurement of socio-economic status in families of children with cancer in Guatemala. Pediatr Blood Cancer 61(11): 2071-2073.

16. Navarrete M, Rossi E, Brivio E, Carrillo JM, Bonilla M, et al. (2014) Treatment of childhood acute lymphoblastic leukemia in central America: a lower-middle income countries experience. Pediatr Blood Cancer 61(5): 803-809.
ISSN: 2574-1241

DOI: 10.26717/BJSTR.2019.17.003063

Nguyen Huu Son. Biomed J Sci \& Tech Res

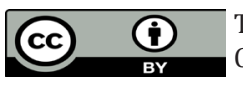

This work is licensed under Creative Commons Attribution 4.0 License

Submission Link: https://biomedres.us/submit-manuscript.php

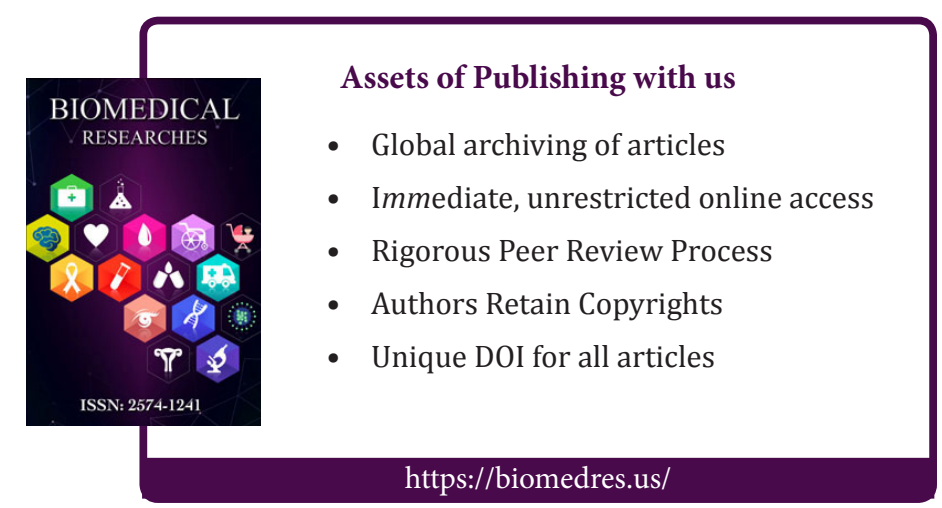

